\title{
Estandarización de la documentación de los procesos de las empresas de Bogotá D.C, en la norma ISO 9001:2008 con cambios a 2015
}

Standardization of documentation
for business processes in Bogotá
D.C., on ISO $9001: 2008$ including the
changes to 2015

Standardisation de la documentation et des processus ISO 9001:2008 (incluant les changements 2015) pour les entreprises de Bogotá appliquant la norme

Padronização da documentação dos processos da empresas de Bogotá DC, de acordo as normas ISO 9001: $2008 \mathrm{com}$ mudanças a 2015

Gerardo Avendaño* Martín Díaz**

Fecha de recepción: 9 de febrero Fecha de aprobación: 8 de marzo Pp. 7-30

* Postdoctorado Penn State University en Manufacturing Engenniering; doctorado Universidad Politécnica de Valencia en Métodos Estadísticos Avanzados; especialización en Ingeniería de Producción, Universidad Distrital Francisco José de Caldas; ingeniero químico, Universidad Nacional de Colombia. Profesor titular, Universidad EAN.

** Ingeniero industrial; especialista en Producción. Docente Corporación Universitaria Minuto de Dios, Facultad de Ciencias Empresariales. 


\section{RESUMEN}

En el presente trabajo de investigación se realizó un estudio de la documentación de los procesos en empresas de la ciudad de Bogotá D.C, con base en los requisitos de la norma ISO 9001:2008 con cambios a 2015. S se realizó un diagnóstico para determinar los lineamientos requeridos de los procesos y las directrices organizacionales, y definir los pasos de la implantación de un Sistema de Gestión de la Calidad.

Se identificaron las motivaciones y preferencias de los empresarios de las organizaciones del sistema de gestión de calidad en una muestra estadísticamente significativa de 60 empresas, en donde se determinó que, para iniciar actividades de mejoramiento, el $77 \%$ tendrán en cuenta mejorar el servicio al cliente y el $31 \%$ de los empresarios prefieren el enfoque basado en procesos en una organización en los cambios de la ISO 9001:2008 a la ISO 9001: 2015.

\section{Palabras Clave}

Estandarización, gestión, procesos, mejoramiento y calidad.

\section{AbStRact}

In this research, a study of the documentation for processes in companies that are located in Bogotá D.C was conducted, based on the requirements of ISO 9001: 2008 including the changes to 2015. A diagnosis to determine the required regulations and organizational guidelines, and to define the steps for the implementation of a quality management system was also performed.

The motivations and preferences of business organizations in the quality management system were identified in a statistically significant sample of 60 companies, where it could be determined that, in order to start improvement activities, $77 \%$ will have to consider upgrading their customer service, and $31 \%$ of employers prefer an approach that is based on the processes in an organization and in the changes from ISO 9001: 2008 to ISO 9001: 2015.

\section{KEYWORDS}

Standardization, management, processes, improvement and quality. 


\section{RÉSUMÉ}

Ce travail d'investigation étudie la documentation des processus des entreprises de Bogotá D.C sur la base des conditions requises par la norme ISO 9001:2008 incluant les changements 2015. Un diagnostic a été mis à jour pour déterminer les critères requis de standardisation des processus et des directives organisationnelles et pour définir les étapes de la mise en place d'un système de Gestion de la Qualité.

Les motivations et préférences des entrepreneurs des organisations du système de gestion de la qualité ont été regroupées dans un échantillon statistiquement significatif de 60 entreprises. Pour commencer les activités d'amélioration, il a été déterminé que $77 \%$ des entreprises devraient améliorer le service client et $31 \%$ d'entre elles passer de la norme ISO 9001:2008 à la norme ISO 9001 : 2015.

\section{MotS CLEFS}

Standardisation, gestion, processus, amélioration et qualité.

\section{ResUMO}

No presente trabalho de pesquisa foi realizado um estudo da documentação dos processos em empresas de Bogotá $D C$, com base nos requisitos da norma ISO 9001: 2008 com mudanças para 2015. Um diagnóstico foi realizado para determinar as orientações necessários dos processos e diretrizes organizacionais e definir as etapas da implementação de um sistema de gestão da qualidade.

Se identificaram as motivações e preferências dos empresarios das orgnizacoes do sistema de gestao de qualidade em uma amostra estatisticamente significativa de 60 empresas, onde foi determinado que, para começar as atividades de melhoria, $77 \%$ consideraram melhorar o serviço ao cliente e $31 \%$ dos empresários preferem o enfoque badeado em processos em uma organização nas de acordo as mudancas da ISO 9001: 2008 para a ISO 9001: 2015.

\section{Palavras-chave}

Padronização, gestão, processos e melhoria da qualidade. 


\section{Introducción}

- I mejoramiento continuo que se debe hacer en los procesos, la exigencia de los clientes en productos y servicios así como el grado de competitividad de las organizaciones, está originando un avance de la gestión de la calidad y la incorporación de nuevos componentes de gestión que permiten adaptarse a dichos cambios. El estándar de calidad ISO 9001:2008 describe un sistema de gestión de calidad aplicable a todo tipo de organización con los nuevos cambios dados en la ISO 9001: 2015, como una herramienta para promover y originar una cultura de calidad en los procesos, procedimientos y actividades de las empresas. La gestión de calidad se ha convertido en un requerimiento indispensable para la estabilidad de las empresas dando prioridad a las necesidades de los clientes y permitiendo dar al comercio productos y servicios con la calidad solicitada..

Las empresas de la ciudad de Bogotá D.C, en la gestión de calidad, tienen una deficiente administración, mal sistema de control, manejo ineficiente de los recursos, deficiencias estructurales y resistencia al cambio. Sin embargo, algunas organizaciones tienen procesos administrativos de direccionamiento estratégico y de apoyo, pero no tienen definidos estos procesos y los ya establecidos, no están establecidos bajo ninguna metodología, no tienen revisado ni aprobado las fechas de emisión, consecuentemente no se les hace revisión ni seguimiento y presentan una débil estandarización aunque estén certificadas con ISO 9001:2008.

Se establece que se debe estandarizar la documentación de los procesos de las diferentes empresas de la ciudad de Bogotá D.C, con base en los requisitos de la norma ISO 9001:2008 con cambios a 2015 haciendo un diagnóstico 
de la situación de los procesos referentes, acordando los lineamientos requeridos de los procesos y las directrices organizacionales; así mismo, definiendo los pasos de la implantación de un sistema de gestión de la calidad.

Es así como se realiza la revisión de los requerimientos de la norma técnica ISO 9001:2008 y su actualización con la versión 2015 además del diagnóstico de la organización para la implementación efectiva en el mejoramiento continuo de las organizaciones

Finalmente, se concluye que el objetivo de esta investigación sobre la documentación de los procesos de las diferentes empresas de la ciudad de Bogotá D.C, con base en los requisitos de la norma ISO 9001:2008 con cambios a 2015, es mantener la eficiencia de la normativa frente a los cambios en las ideologías y tendencias de gestión en el mundo y los posibles cambios que representan los avances tecnológicos en el futuro.

Uno de los aportes fundamentales que la revisión de la norma ISO 9001:2015 ha traído consigo es el enfoque desde el que se debe definir el contexto de la organización y la consideración que se debe hacer de las partes interesadas en la organización, cuya importancia radica en el hecho de que la detección de los factores pertinentes a la planificación e implementación de un sistema de gestión de la calidad está estrechamente vinculado con la consecución de los resultados previstos (González, 2016). 


\section{Metodología}

Con base en la revisión bibliográfica relacionada en la norma ISO 9001:2008, con cambios a la ISO 9001: 2015, se realizó el trabajo de investigación en donde se empleó un tipo de estudio de carácter descriptivo, que consiste en llegar a conocer las situaciones a través de la descripción exacta de las actividades la cual se desarrolla mediante una solución al planteamiento del problema y los datos descriptivos que se expresan en términos cualitativos y cuantitativos. Los estudios cualitativos contribuyen a identificar los factores importantes que deben ser medidos; los estudios cuantitativos pueden medir las diferentes unidades, elementos o categorías identificables.

El trabajo de investigación está encaminado específicamente en las empresas de manufactura y de servicios de la ciudad de Bogotá D. C. que están en procesos de implementación, incluidos extensamenteen la autoevaluación y revisión o certificación evaluada por un auditor o grupo de auditores que se ajustan a los requisitos del sistema o modelo de gestión de calidad; organizaciones que tienen grandes dificultades para establecer y aplicar un sistema de gestión de calidad debido a una deficiente administración, mal sistema de control, manejo ineficiente de los recursos, deficiencias estructurales y resistencia al cambio y a los procesos existentes; no están enmarcadas bajo ninguna metodología, no tienen revisado ni aprobadas las fechas de emisión, por lo tanto se debe establecer una estandarización de la norma ISO 9001:2008 con cambios a la ISO 9001: 2015. La investigación cualitativa se hace mediante los métodos de recolección de la información en donde se establecen las investigaciones de publicaciones, datos estadísticos y artículos que permitan definir los objetivos definidos y el desarrollo del contenido. 
Inicialmente se analizó en qué medida las organizaciones estudiadas están certificadas con el sistema de gestión de calidad, por medio de encuestas que se ajustan a los requisitos de la norma. De esta forma, conocer la viabilidad de la gestión actual de las empresas estudiadas bajo la norma ISO 9001:2008 con cambios a la ISO 9001: 2015.

Luego, mediante el análisis detallado a una muestra estadísticamente obtenida a 60 empresas, se determinaron las motivaciones y preferencias de los empresarios de las organizaciones del sistema de gestión de calidad y se permitió conocer las actividades, acciones de los procesos y los problemas asociados a las mismas, los puntos fuertes y débiles con respecto a los requisitos de la norma ISO.

Con la realización del diagnóstico inicial y su análisis respectivo posteriormente, se pudo conocer el punto de partida para el diseño del estudio que servirá como referencia para la estandarización, ya que se visitó cada una de las empresas y se les aplicó a los encargados de los procesos, las encuestas, con el fin de encontrar el nivel de cumplimiento de cada uno de los requisitos, los documentos y registros que son obligatorios por la norma técnica y los necesarios por la organización. Reflejaron de forma realista, la situación actual de las empresas con relación a los principios de la gestión de la calidad, los requisitos de la norma ISO 9001:2008 y los cambios en la ISO 9001:2015. 


\section{Resultados}

- I resultado de la investigación, en cuanto al aporte del Canálisis de validación del instrumento de la encuesta de las empresas de la ciudad de Bogotá D.C, y a la gestión de calidad, en donde se identificaron las motivaciones y preferencias de los empresarios de las organizaciones, establece que el $33 \%$ de estas son grandes, el $40 \%$ medianas, y están mejor encaminadas, estructuradas en la administración, control y manejo de los recursos, y cambio en la cultura organizacional. Por su parte, el $27 \%$ corresponde a las pequeñas empresas, en donde se deduce que se deben iniciar actividades de mejoramiento así: sobre el $77 \%$ en temas del servicio al cliente.

El $72 \%$ de las empresas encuestadas que tienen el sistema de gestión de calidad, perciben que para la creación de un producto o servicio es necesario tener en cuenta las necesidades de los clientes; el $68 \%$ de las empresas creen en la importancia de la calidad percibida del producto o servicio.

El total de las empresas intervenidas deben tener en cuenta los siguientes aspectos:

- La alta dirección debe incluir en los sistemas y procesos, la eficiencia y eficacia para que repercuta en la satisfacción de los clientes.

- Evaluar la efectividad de la operación mediante el control de los procesos utilizando métodos tales como autoevaluaciones y revisiones por parte de la dirección.

- Implementar las acciones necesarias para alcanzar los resultados planificados de las organizaciones y la mejora continua de los procesos. Para ello, realizar los 
procedimientos y las actividades de gestión mediante las provisiones de los recursos para la realización del producto con mediciones posteriores y que se puedan determinar los registros pertinentes; establecer, implementar y mantener elSGC apoyando la operación en sus procesos, soportándose en la documentación de tal forma que se satisfagan los requisitos contractuales, legales y reglamentarios.

La documentación actual de las organizaciones cumple con las necesidades y expectativas de los clientes, partes interesadas y al interior de la organización, ya que la generación, uso y control de la documentación es funcional y permite el acceso a los clientes y proveedores que lo requieran.

Las entidades cuentan con los documentos necesarios para asegurar su eficaz planificación, operación y control de procesos, como el manual de calidad, que ha permitido el desempeño enérgico por parte de los servidores en cuanto al cumplimiento de los procedimientos documentados para la estandarizar de la gestión de las operaciones, evitando los reprocesos y la reducción del producto no conforme.

Los procedimientos de control de documentos han facilitado la aprobación, revisión y mantenimiento de los documentos de la entidad y la distribución de los documentos de origen externo, el almacenamiento, protección, tiempo de retención y disposición de los mismos.

Los registros en las empresas proporcionan evidencia de la conformidad con los requisitos del producto y/o servicio, del SGC que ha contribuido a la mejora continua a través de la comprensión de las necesidades y expectativas actuales y futuras de los clientes. Así mismo, genera el compromiso, por parte de los servidores, al uso de sus habilidades para el beneficio de la entidad, en cuanto a las actividades y recursos gestionados en los procesos; permite el cumplimiento de la 
planificación de forma más eficiente con la interrelación de los procesos dentro del sistema; contribuye al logro de los objetivos con las mediciones del desempeño de los procesos y procedimientos para determinar si se han alcanzado los objetivos planificados, las relaciones con los proveedores y los convenios con otras entidades.

Los macroprocesos determinados por la entidad misionales, de apoyo, estratégicos y de medición-, han permitido el desempeño y cumplimiento de los objetivos institucionales y de calidad; las acciones de verificación de los procesos para establecer acciones correctivas, preventivas o mejoras necesarias para la medición, recopilación y validación de datos para la toma de decisiones además de mirar el desempeño institucional.

Con el proceso de auditoría interna, se ha permitido el establecimiento de las fortalezas y debilidades del SGC para su mejora, logrando una evaluación de eficacia, eficiencia y efectividad de la entidad hacia las acciones encaminadas a la eliminación de las no conformidades y a través de un análisis de causas; así, la evaluación del desempeño frente a los planes, objetivos y metas, y la identificación de áreas de mejora acorto plazo o proyectos de mejora, continua a largo plazo, con acciones correctivas y preventivas que permiten el control en la ocurrencia de las no conformidades.

Los cambios de la ISO 9001:2008 a la ISO 9001: 2015 que se han evidenciado en las organizaciones analizadas, se tendrán en cuenta así:

- El 31\% prefieren el enfoque basado en procesos en una organización.

- El 23\% prefieren el otros, es decir, mejor redacción de la norma para hacerla más general y entendible a empresas 
de servicios; la planificación y el liderazgo como ejes importantes; los términos documento y registro se han sustituido ambos; información documentada; del cliente a las partes interesadas: incluye a accionistas, personas, sociedad, proveedores, alianzas con las expectativas y necesidades; los auditores se van a poner mucho más estrictos.

- El $18 \%$ prefieren las acciones para abordar los riesgos y las oportunidades.

- El 15\% prefieren que haya desaparecido el término acción preventiva.

- El $13 \%$ prefieren que se haga la realización de producto a operaciones.

\section{Conclusiones}

Ce concluye que se debe elaborar una guía de Sestandarización de la documentación de los procesos de las diferentes empresas de la ciudad de Bogotá D.C, con base en los requisitos de la norma ISO 9001:2008 con cambios a 2015. Esto ayudará a simplificar la toma de decisiones, se mejorará la calidad de los procesos de manera efectiva, se avanzará hacia la sostenibilidad y el aseguramiento de la calidad, minimizando el riesgo ya que, al consultar a los empresarios y hacer un diagnóstico, se estableció que:

- El $23 \%$ de las organizaciones encuestadas tendrán que mejorar las actividades de los procesos para dar un mejor servicio al cliente, teniendo lineamientos de mejora continua en el desempeño de las entidades en donde 
debe ser un objetivo permanente que se verá reflejado en la simplificación administrativa para modificar la organización, teniendo en cuenta los elementos del proceso con relación al ciclo Planear, Hacer, Verificar y Actuar (PHVA) y la actualización de los documentos en los macroprocesos estratégicos, misionales y de apoyo, además de los manuales de gestión de procesos y procedimientos.

- El $28 \%$ de las organizaciones encuestadas creen que deben mejorar las actividades en torno a la creación de un producto según las necesidades del cliente, ya que necesitan definir quiénes son sus clientes, precisar cuáles son los factores de éxito con los que pueden responder a las necesidades, requerimientos y expectativas, y establecer los procesos efectivos para generar productos y servicios; enfocar sus esfuerzos en mejorar sus procesos y desarrollar métodos y evaluarlos teniendo en cuenta que hay necesidades de orden mayor o secundarias que el cliente tiene y que deben satisfacerse.

- El 32\% de las organizaciones encuestadas creen que deben mejorar la calidad del producto o servicio para que sea excelente, en donde se debe enfocar y tener directrices dadas por la alta dirección para desarrollar conciencia en todos los integrantes de la organización dirigida hacia la calidad; esta debe estar alineada con la misión y la visión de la empresa, generando y manteniendo un ambiente interno favorable en el cual todos puedan llegar a involucrarse en el logro de los objetivos establecidos

- El $100 \%$ de las organizaciones han implementado acciones necesarias para alcanzar los resultados planificados, pero les falta integrar políticas y objetivos para la obtención de resultados. 
La mejor estrategia es involucrar, desde el comienzo, a todos los empleados y contratistas en la construcción de la documentación del sistema de gestión de la calidad en cuanto a procesos, procedimientos, guías y formatos, ya que son ellos quienes conocen la manera como se llevan a cabo las actividades; son quienes, en última instancia, van a aplicar las disposiciones que se documenten. Para ello, se conforman equipos de trabajo por proceso en los que se elaboran los documentos que luego serán sometidos a revisión por otras instancias técnicas y/o de dirección.

El $100 \%$ de las organizaciones han determinado tener los registros pertinentes para establecer, implementar y mantener el sistema de gestión de calidad (SGC) y apoyar la operación eficaz y eficiente de sus procesos, pero les falta tener mejores prácticas en los sistemas incorporados.

El $88 \%$ de las organizaciones no han desarrollado estudios de comparación con otras entidades nacionales o internacionales en busca de la mejora continua, ya que algunas empresas trabajan con modelos diferentes como: EFQM, Deming, SeisSigma, Kaizen, 7 Pasos, Crosby, entre otros.

El $100 \%$ de las organizaciones han mejorado los procesos y procedimientos documentados permitiendo la estandarización de la gestión de las entidades encuestadas, evitando los reprocesos y la reducción del producto no conforme; sin embargo, falta la guía general, ya que algunas han adoptado por la metodología BPMN para la caracterización y documentación de procesos, entre otras.

Después de realizar un diagnóstico en la organización para implementar cualquier sistema de gestión, se deben tener en cuenta los debe de la norma que se requieran implementar, ya que estos son los mínimos requisitos que se necesitan cumplir en la ejecución y diagnóstico de la norma ISO 9001:2008 con cambios a 2015. 
Los pasos de la Implantación de un Sistema de Gestión de la Calidad de las diferentes empresas de la ciudad de Bogotá D.C, de la norma ISO 9001:2008 con cambios a 2015 son:

- Diagnóstico de la organización en el sistema de gestión de calidad ISO 9001: se debe establecer un diagnostico al sistema de gestión de calidad ISO 9001: 2008 con cambios a 2015, teniendo en cuenta en la organización los objetivos de un sistema de calidad; la satisfacción del cliente, obtención de nuevos clientes, organización sistemática de la empresa en gestión por procesos, mejora continua, emular o diferenciarse de la competencia, reducir los costos de no calidad. Tener en cuenta el requisito de las administraciones y exigencias de grupo multinacional y los principios básicos de la gestión de calidad en la organización orientada al cliente; liderazgo, implicación del personal, enfoque por procesos, dirección basada en sistemas y mejora continua y filosofía. La organización debe estar enfocada al cliente, identificando las necesidades y expectativas del mismo, convirtiéndolas en requisitos para alcanzar su satisfacción con un enfoque basado en los procesos y con la finalidad de que se concentre la atención en el resultado de cada uno de los procesos en lugar de las tareas; así mismo, establecer la mejora continua en la organización.

- Consultar la norma ISO 9001 introductoria: mirar la norma ISO 9001:2008 con cambios a 2015 de cómo está estructurada, en donde los cuatro primeros temas son de carácter introductorio basándose en declaraciones de principios, estructura y descripción de la empresa, requisitos generales, etc.; así, tener en cuenta los cambios en el capítulo uno, de objeto y campo de aplicación, de generalidades y aplicación, a alcance. En el capítulo dos, estudiar las referencias normativas; en el tercer capítulo, términos y definiciones; en el capítulo cuatro, ver el sistema de gestión de calidad de los requisitos generales 
y los requisitos de la documentación; ver el contexto de la organización, comprender su contexto; determinar las necesidades y expectativas de las partes interesadas, determinar el alcance del sistema de gestión de la calidad y sus procesos.

- Consultar la norma ISO 9001 en cuanto a procesos o documentos a implementar: mirar cómo está estructurada, a partir de los capítulos cinco al ocho los cuales están orientados a procesos, y en ellos se agrupan los requisitos para la implantación del sistema de calidad teniendo en cuenta los cambios en el capítulo cinco; sobre el compromiso de la dirección en cuanto al liderazgo, y del enfoque al cliente hacia la política de la organización; de la política de calidad a las funciones de la organización, responsabilidades y autoridades, y la planeación, responsabilidad, autoridad y comunicación, y revisión por la dirección, en donde la norma ISO 9001: 2015 no aplica. Revisar, en el capítulo seis de gestión de los recursos, de provisión de recursos, recursos humanos, infraestructura y ambiente de trabajo, con cambios a la planificación en acciones para hacer frente a los riesgos y oportunidades, objetivos de la calidad y la planificación para alcanzarlos y planificación de los cambios. En el capítulo siete, de realización del producto en la planificación de su realización, procesos relacionados con el cliente, diseño y desarrollo, compras, producción y prestación del servicio; control de los equipos de seguimiento y control con cambios a la norma ISO 9001: 2015, a soporte en recursos, competencia, conciencia, comunicación e información documentada. En el capítulo ocho, de medición análisis y mejora; en generalidades, seguimiento y medición, control del producto no conforme, análisis de datos y mejora con cambios a la norma ISO 9001:2015, a funcionamiento en planificación y control operacional, requisitos para 
productos y servicios, diseño y desarrollo de productos y servicios, control de los procesos externamente proporcionados, productos y servicios, producción y prestación del servicio, lanzamiento de productos y servicios y control de las salidas no conformes. En la nueva norma ISO 9001:2015, se debe hacer la evaluación del rendimiento en monitoreo, medición, análisis y evaluación en la auditoría interna y gestión de la revisión; por último, se deben realizar mejoras, en generalidades.

- Comparación e implementación: comparación de la norma ISO 9001 de procesos o documentos a implementar con la organización y a analizar, teniendo en cuenta las metas en cómo, cuándo -tiempo-, y donde-lugar-.

- Conclusiones y recomendaciones: se debe elaborar un informe de los asesores o consultores a la gerencia o accionistas, sobre los cambios establecidos. 


\section{Referencias bibliográficas}

González, H. (2016). Transición a ISO 9001:2015. Consideraciones. Calidad y gestión. Recuperado de: https://calidadgestion.wordpress.com/tag/nueva-iso9001-version-2015/

The British Standards Institution. (2016). Pasando de ISO 9001:2008 a ISO 9001:2015. El nuevo estándar internacional para los sistemas de gestión de la calidad. Recuperado de: http://www.bsigroup.com/LocalFiles/ esES/Documentos \%20tecnicos/Revisiones \% 201SO/ ISO\%209001/ISO-9001 guia\%20de\%20transicion.pdf. 


\section{Anexos}

\section{VICERRECTORÍA DE INVESTIGACIÓN- PROYECTO}

"Guía de estandarización de la documentación de los procesos de las diferentes empresas de la ciudad de Bogotá D.C, con base en los requisitos de la norma ISO 9001:2008 con cambios a 2015"

\section{ENCUESTA APLICADA A LAS DIFERENTES EMPRESAS DE BOGOTÁ D.C.}

Nombre del encuestador:

$N^{\circ}$. de encuestador:

Nombre del encuestado:

$\mathrm{N}^{\circ}$. de encuesta:

\section{Presentación del encuestador}

Buenos días/tardes,

Mi nombre es

La presente entrevista se aplica con el objetivo de realizar una exploración sobre el nivel de conocimiento de la documentación estandarizada de los diferentes procesos de las empresas de la ciudad de Bogotá D.C, con base en los requisitos de la norma ISO 9001:2008 con cambios a 2015, para ser analizado para efectos académicos, garantizando la total reserva y el buen manejo de la información obtenida.

Estamos interesados en conocer su opinión, por favor, ¿sería tan amable de contestar el siguiente cuestionario? Gracias.

\section{Datos generales}

Fecha:

Nombre de la empresa:

Año de creación: Teléfonos:

e-mail:

Clasificación de la empresa por activos o por número de empleados: Grande $>200$ Mediana $200<$ Pequeña $50<$

Micro $10<$ 


\section{CONOCIMIENTO E INTERÉS EN LA CALIDAD}

1. Dentro de las siguientes opciones, clasifique de 1 a 5 , siendo 5 la más importante, la que lo motivaría a iniciar actividades en mejoramiento de la calidad en los procesos

a. Mejorar el servicio al cliente

b. Presiones legales o ambientales

c. Aumento en los ingresos

d. Responsabilidad social

e. Razones de competitividad

f. Otro

2. De las siguientes actividades, señale cuál es la condición más importante que tiene en cuenta al crear un producto o servicio en la empresa

a. Necesidad de los clientes

b. cambio de presentación

c. Por moda

d. por innovación

e. por una nueva innovación

f. otro

3. ¿Cómo usted califica los parámetros de conformidad en la empresa, para la elaboración de un producto o servicio?

a. Parámetros del cliente

b. Producto garantizado

c. producto de precio asequible

d. Cumplir con las normas ISO

e. Producto más resistente

e. Otro cuál?

4. ¿Cómo usted califica la calidad percibida del producto o servicio, en la empresa?

a. Excelente calidad

b. Supera las expectativas

c. Se puede recomendar por su calidad

d. Excelente presentación

e. Imagen impecable

e. Otro cuál? 


\begin{tabular}{|c|c|c|c|}
\hline ITEM & SI & NO & POR QUÉ \\
\hline $\begin{array}{l}\text { 5. La alta dirección ha definido sistemas y } \\
\text { procesos comprensibles, gestionables y } \\
\text { mejorables en lo referente a eficacia y } \\
\text { eficiencia, para la satisfacción de los clientes }\end{array}$ & & & \\
\hline $\begin{array}{l}\text { 6. La alta dirección se asegura de evaluar } \\
\text { la eficacia y eficiencia de la operación, } \\
\text { mediante el control de los procesos. }\end{array}$ & & & \\
\hline $\begin{array}{l}\text { 7. La alta dirección se asegura de evaluar } \\
\text { la eficacia y eficiencia de la operación, } \\
\text { mediante las medidas y datos utilizados } \\
\text { para determinar el desempeño satisfactorio } \\
\text { de la entidad. }\end{array}$ & & & \\
\hline $\begin{array}{l}\text { 8. La entidad utiliza métodos para evaluar } \\
\text { la mejora de los procesos, tales como } \\
\text { autoevaluaciones y revisiones por parte de } \\
\text { la dirección. }\end{array}$ & & & \\
\hline $\begin{array}{l}\text { 9. La entidad ha implementado acciones } \\
\text { necesarias para alcanzar los resultados } \\
\text { planificados y la mejora continua de los } \\
\text { procesos. }\end{array}$ & & & \\
\hline $\begin{array}{l}\text { 10. La descripción de los procesos de la entidad } \\
\text { se incluyen las actividades de gestión, la } \\
\text { provisión de recursos, la realización del } \\
\text { producto y las mediciones posteriores. }\end{array}$ & & & \\
\hline $\begin{array}{l}\text { 11. La entidad ha determinado los registros } \\
\text { pertinentes para establecer, implementar, } \\
\text { mantener el SGC y apoyar la operación } \\
\text { eficaz y eficiente de sus procesos. }\end{array}$ & & & \\
\hline $\begin{array}{l}\text { 12. La naturaleza y extensión de la docu- } \\
\text { mentación satisface los requisitos } \\
\text { contractuales, legales y reglamentarios }\end{array}$ & & & \\
\hline $\begin{array}{l}\text { 13. La documentación en la entidad satisface } \\
\text { las necesidades y expectativas de los } \\
\text { clientes, partes interesadas y al interior de } \\
\text { la misma. }\end{array}$ & & & \\
\hline $\begin{array}{l}\text { 14. La generación, uso y control de la docu- } \\
\text { mentación es funcional y permite el } \\
\text { acceso a los clientes, proveedores y otras } \\
\text { partes interesadas. }\end{array}$ & & & \\
\hline
\end{tabular}




\begin{tabular}{|c|c|c|c|}
\hline ITEM & SI & NO & POR QUÉ \\
\hline $\begin{array}{l}\text { 15. La entidad cuenta con los documentos } \\
\text { necesarios para asegurar su eficaz plani- } \\
\text { ficación, operación y control de procesos. }\end{array}$ & & & \\
\hline $\begin{array}{l}\text { 16. La entidad ha desarrollado estudios } \\
\text { de comparación con otras entidades } \\
\text { nacionales o internacionales en busca de } \\
\text { la mejora continua. }\end{array}$ & & & \\
\hline $\begin{array}{l}\text { 17. El Manual de Calidad ha permitido el } \\
\text { desempeño eficaz por parte de los servi- } \\
\text { dores en cuanto al cumplimiento de los } \\
\text { procedimientos documentados. }\end{array}$ & & & \\
\hline $\begin{array}{l}\text { 18. Los procesos y procedimientos documen- } \\
\text { tados han permitido estandarizar la gestión } \\
\text { de la entidad, evitando los reprocesos y la } \\
\text { reducción del producto no conforme. }\end{array}$ & & & \\
\hline $\begin{array}{l}\text { 19. El procedimiento control de documentos ha } \\
\text { facilitado la aprobación, revisión y mante- } \\
\text { nimiento de los documentos de la entidad. }\end{array}$ & & & \\
\hline $\begin{array}{l}\text { 20. El procedimiento control de documentos } \\
\text { ha facilitado, la identificación, control y } \\
\text { distribución de los documentos de origen } \\
\text { externo. }\end{array}$ & & & \\
\hline $\begin{array}{l}\text { 21. El control de los registros en la entidad } \\
\text { ha facilitado la identificación, almace- } \\
\text { namiento, protección, tiempo de retención } \\
\text { y disposición de los mismos. }\end{array}$ & & & \\
\hline $\begin{array}{l}\text { 22. Los registros en la entidad proporcionan } \\
\text { evidencia de la conformidad con los } \\
\text { requisitos del producto y/o servicio. }\end{array}$ & & & \\
\hline $\begin{array}{l}\text { 23. EI SGC ha contribuido a la mejora } \\
\text { continua a través de la comprensión de } \\
\text { las necesidades y expectativas actuales y } \\
\text { futuras de los clientes. }\end{array}$ & & & \\
\hline $\begin{array}{l}\text { 24. El SGC ha generado el compromiso por } \\
\text { parte de los servidores en cuanto al uso } \\
\text { de sus habilidades para el beneficio de la } \\
\text { entidad. }\end{array}$ & & & \\
\hline
\end{tabular}




\begin{tabular}{|c|c|c|c|}
\hline \multirow{2}{*}{\begin{tabular}{|l|} 
ITEM \\
25. Las actividades y recursos gestionados en los \\
procesos han permitido el cumplimiento \\
de la planificación de forma más eficiente
\end{tabular}} & SI & NO & POR QUÉ \\
\hline & & & \\
\hline \multicolumn{4}{|l|}{$\begin{array}{l}\text { 26. La interrelación de los procesos como un } \\
\text { sistema ha contribuido al logro de los } \\
\text { objetivos de forma eficaz y eficiente. }\end{array}$} \\
\hline \multicolumn{4}{|l|}{$\begin{array}{l}\text { 27. Las mediciones del desempeño de los } \\
\text { procesos, han permitido determinar si se } \\
\text { han alcanzado los objetivos planificados. }\end{array}$} \\
\hline \multicolumn{4}{|l|}{$\begin{array}{l}\text { 28. Las relaciones con los proveedores y } \\
\text { los convenios con otras entidades ha } \\
\text { permitido mejorar la eficacia y eficiencia } \\
\text { de los procesos. }\end{array}$} \\
\hline \multicolumn{4}{|l|}{$\begin{array}{l}\text { 29. La operación e interacción de los procesos } \\
\text { ha permitido que la entidad tenga la } \\
\text { capacidad de satisfacer los requisitos de } \\
\text { sus clientes y partes interesadas. }\end{array}$} \\
\hline \multicolumn{4}{|l|}{$\begin{array}{l}\text { 30. Los procesos determinados por la entidad } \\
\text { (misionales, apoyo, estratégicos y de } \\
\text { medición) han permitido el desempeño y } \\
\text { cumplimiento de los objetivos institucio- } \\
\text { nales y de calidad. }\end{array}$} \\
\hline \multicolumn{4}{|l|}{$\begin{array}{l}\text { 31. Las acciones de verificación de los procesos } \\
\text { han permitido establecer acciones correcti- } \\
\text { vas, preventivas o mejoras necesarias para } \\
\text { la eficacia y eficiencia de los mismo. }\end{array}$} \\
\hline \multicolumn{4}{|l|}{$\begin{array}{l}\text { 32. La medición, recopilación y validación de } \\
\text { datos y el uso previsto de los mismos le han } \\
\text { permitido a la entidad asegurar la toma de } \\
\text { decisiones y el desempeño institucional. }\end{array}$} \\
\hline \multicolumn{4}{|l|}{$\begin{array}{l}\text { 33. El proceso de auditoría interna ha permitido } \\
\text { el establecimiento de las fortalezas y } \\
\text { debilidades del SGC para su mejora. }\end{array}$} \\
\hline $\begin{array}{l}\text { 34. La auditoría interna ha permitido la } \\
\text { evaluación de la eficacia, eficiencia y } \\
\text { efectividad de la entidad. }\end{array}$ & & & \\
\hline
\end{tabular}




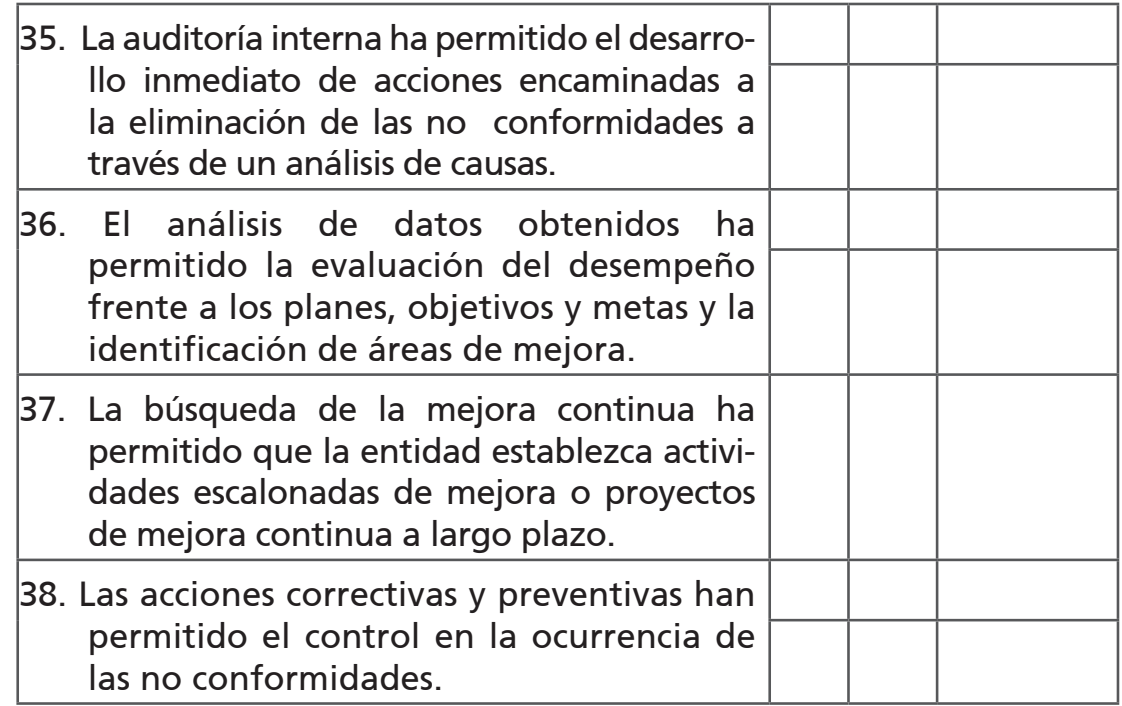

39. Clasifique de 1 a 5 (siendo 5 la más importante) los cambios de la ISO 9001:2008 a la ISO 9001: 2015, que se han evidenciado en su organización según las siguientes opciones

a. Realización de Producto a Operaciones

b. Enfoque basado en procesos en una organización

c. Desaparece el término Acción Preventiva

d. Acciones para abordar los riesgos y las oportunidades

e. . Otros

40. ¿Desea usted adicionar una información diferente y que no esté dentro de la encuesta?

Muchas gracias por su amabilidad y por el tiempo dedicado a contestar esta encuesta 